\title{
The prognostic significance of percentage of tumour involvement according to disease risk group in men treated with radical prostatectomy
}

\author{
Jong Jin $\mathrm{Oh}^{1}$, Sang Cheol Lee ${ }^{1}$, Chang Wook Jeong ${ }^{1}$, Cheol Yong Yoon ${ }^{1}$, Seong Jin Jeong ${ }^{1}$, Sung Kyu Hong ${ }^{1}$, \\ Seok-Soo Byun ${ }^{1}$, Yoon Kwan Rho' ${ }^{1}$, Gheeyoung Choe $^{2}$ and Sang Eun Lee ${ }^{1}$
}

We investigated the prognostic significance of percentage of tumour involvement (PTI) according to the clinicopathological features of prostate cancer among patients who underwent radical prostatectomy (RP). A retrospective study of 534 patients who underwent RP between September 2003 and March 2008 without any neoadjuvant or adjuvant therapy was performed. The associations of PTI with various clinicopathological features and biochemical recurrence-free survival were examined via uni- and multivariate analyses. The predictive accuracy of the multivariate model was assessed with a receiver operating characteristics-derived area under the curve. PTI was demonstrated to be significantly associated with preoperative prostate-specific antigen (PSA) level $(P=0.001)$, pathological Gleason score $(P<0.001)$, extraprostatic tumour extension $(P<0.001)$, seminal vesicle invasion $(P<0.001)$ and positive surgical margin $(P<0.001)$ in univariate analyses. When patients were stratified into disease risk groups, PTI was an independent predictor of biochemical recurrence-free survival in multivariate analysis only among the low-risk group $(P=0.033)$ but not the intermediate-

$(P=0.287)$ or the high-risk groups $(P=0.828)$. The addition of the PTI did not significantly increase the accuracy of the multivariate model devised for the prediction of biochemical recurrence-free survival among both total patients $(P=0.459)$ and the low-risk group $(P=0.268)$, respectively. In conclusion, although PTI appeared to be a more significant prognostic factor among patients with low-risk disease than among those with higher risk diseases, overall, the PTI may not provide additional prognostic information beyond what can already be obtained via established prognostic factors.

Asian Journal of Andrology (2011) 13, 828-832; doi:10.1038/aja.2011.111; published online 26 September 2011

Keywords: biochemical recurrence-free survival; percentage of tumour involvement; prognosis; prostate cancer; prostatectomy

\section{INTRODUCTION}

In oncology, tumour burden or size has traditionally been regarded as a useful surrogate for prognosis in various types of malignancies. Previously, some researchers have reported that tumour volume (TV) in radical prostatectomy (RP) specimens, which already demonstrates significant correlations with other established prognostic factors, is independently associated with the progression of prostate cancer. ${ }^{1-5}$ However, other reports have produced contradictory data ${ }^{6-8}$ Currently, the controversy continues in regard to the prognostic value of TV in prostate cancer. Moreover, an accurate assessment of TV is not a readily performed procedure, as it requires whole-mount sectioning and specialized equipment, resulting in added costs. In most centres, TV is not routinely included in the pathological reports on RP specimens.

Percentage of tumour involvement (PTI) is a pathological parameter relatively easy to attain. It requires only the visual estimation of tumour burden on pathological slides and the averaging of results without the need for additional equipment or special specimen handling. As with TV, contradictory data have been reported concerning the prognostic value of PTI in prostate cancer. ${ }^{9-15}$ In examining the ongoing controversies surrounding the prognostic values of TV and PTI in prostate cancer, we hypothesized that the impact of tumour size might differ according to the tumour aggressiveness Thus, we investigated the prognostic significance of the PTI, a routinely reported pathological parameter at our institution, according to the clinicopathological features of disease among patients who underwent RP for prostate cancer.

\section{MATERIALS AND METHODS}

Patients

After obtaining institutional review board approval, we reviewed the data of 563 patients who underwent RP for prostate cancer between September 2003 and March 2008 at our institution and were followed up for more than 2 years postoperatively. After excluding patients who underwent neoadjuvant or adjuvant hormone or radiation therapy and those patients with missing data, a total of 534 patients were included in the final analysis.

${ }^{1}$ Department of Urology, Seoul National University Bundang Hospital, Seongnam, Kyunggi-do 463-707, Korea and ${ }^{2}$ Department of Pathology, Seoul National University Bundang Hospital, Seongnam, Kyunggi-do 463-707, Korea 


\section{Pathological evaluation}

The specimens from RPs were weighed, fixed intact in 20\% neutral buffered formalin and sectioned at 3-4 mm intervals with the apical and bladder neck portion sectioned radially to allow evaluation of the margin status parallel to urethra. For each pathological slide, the percentage of the slide with tumour involvement was estimated, and PTI was determined by averaging the estimates from all slides as previously reported by others ${ }^{12,13}$

\section{Statistical analysis}

The data assessed included patient age, preoperative prostate-specific antigen (PSA) level, prostate specimen weight, PTI, pathological Gleason score, pathological stage, surgical margin status, lymph node involvement and postoperative follow-up PSA data. The association of PTI with other clinicopathological variables was analysed via MannWhitney $U$ or Kruskal-Wallis tests. For our analysis, the total patient population was stratified into three disease risk groups according to preoperative PSA level and pathological Gleason score with consideration of the PTI as a pathological variable. The low-risk group had a preoperative PSA level $<10 \mathrm{ng} \mathrm{ml}^{-1}$ and a pathological Gleason score $\leqslant 6$, the intermediate-risk group had a PSA of $10-20 \mathrm{ng} \mathrm{ml}^{-1}$ and a pathological Gleason score of 7, and the high-risk group had a PSA $>20 \mathrm{ng} \mathrm{ml}^{-1}$ and a pathological Gleason score $\geqslant 8 .{ }^{16}$ Biochemical recurrence was defined as two consecutive rises in PSA of $0.2 \mathrm{ng}$ $\mathrm{ml}^{-1}$ or higher at least 2 months following RP. ${ }^{17}$ The probability of biochemical recurrence-free survival was determined using KaplanMeier curves along with a log-rank test used to assess statistical significance. The effects of the various variables on biochemical recurrence-free survival were assessed using a Cox proportional hazards model. The predictive accuracy of the multivariate logistic regression model was assessed with a receiver-operating characteristics-derived area under the curve. The areas under the curve were compared via a Mantel-Haenszel test. The SPSS software package version 15.0 (SPSS Inc., Chicago, IL, USA) was used for all statistical analyses. A twotailed $P<0.05$ was considered significant for all analyses.

\section{RESULTS}

The patient characteristics are listed in Table 1 . After stratification by disease risk, 110 (20.6\%) patients had low-risk disease, 315 (59.0\%) had intermediate-risk disease and 109 (20.4\%) had high-risk disease. Overall, biochemical recurrence was observed in 105 (19.7\%) patients during the mean follow-up period of $51.2 \pm 13.5$ months. The biochemical recurrence-free survival was higher in patients with low-risk disease than those with intermediate- or high-risk disease (log-rank $P<0.0001)$

Among all patients, PTI was significantly associated with preoperative PSA level $(P=0.001)$, pathological Gleason score $(P<0.001)$, extraprostatic extension of tumour $(P<0.001)$, seminal vesicle invasion $(P<0.001)$ and positive surgical margin $(P<0.001)$ in univariate analyses. Also, the PTI was associated with biochemical recurrencefree survival among the total patient population in univariate analysis (log-rank $P<0.001$ ).

A multivariate Cox proportional hazards regression model indicated that PTI was an independent predictor of biochemical recurrence-free survival $(P=0.042)$ among the total patient population (Table 2).

When patients were stratified into three groups according to disease risk assessment, the patients with a PTI $\geqslant 15 \%$ demonstrated a lower rate of biochemical recurrence-free survival than those with a PTI $<15 \%$ among both the low- (log-rank $P<0.001)$ and intermediate-risk
Table 1 Patient characteristics $(n=534)$

\begin{tabular}{lc}
\hline & Value \\
\hline Age at surgery (mean \pm s.d), years & $64.9 \pm 6.7$ \\
Preoperative PSA (mean \pm s.d.), ng ml & -1 \\
Prostate specimen weight (mean \pm s.d.), g & $11.9 \pm 12.2$ \\
Pathological Gleason score $(n, \%)$ & $40.7 \pm 14.2$ \\
$\quad \leqslant 6$ & \\
7 & $136(25.5)$ \\
$\geqslant 8$ & $339(63.5)$ \\
Extraprostatic tumour extension ( $n, \%)$ & $59(11.0)$ \\
$\quad$ Absent & \\
Present & $366(68.5)$ \\
Seminal vesicle invasion $(n, \%)$ & $168(31.5)$ \\
$\quad$ Absent & \\
Present & $488(91.4)$ \\
Surgical margin $(n$, \%) & $46(8.6)$ \\
$\quad$ Negative & \\
Positive & $334(62.5)$ \\
Biochemical recurrence $(n, \%)$ & $200(37.5)$ \\
$\quad$ No & \\
$\quad$ Yes & $429(80.3)$ \\
Percent of tumour involvement $(n, \%)$ & $105(19.7)$ \\
$<5$ & \\
$5-10$ & $173(32.4)$ \\
$11-15$ & $105(19.7)$ \\
$16-20$ & $68(12.7)$ \\
$>20$ & $38(7.1)$ \\
\hline
\end{tabular}

Abbreviation: PSA, prostate-specific antigen.

groups (log-rank $P=0.001)$, but not among high-risk group (log-rank $P=0.272$ ) in univariate analyses, respectively (Figure 1). In multivariate analyses, the PTI was an independent predictor of biochemical recurrence-free survival after RP only among the low-risk group $(P=0.033)$ but not among the intermediate- $(P=0.287)$ and high-risk groups $(P=0.828)$, respectively (Table 3$)$.

When a multivariate model incorporating age, PSA, prostate volume, pathological Gleason score, extraprostatic extension of tumour, seminal vesicle invasion and surgical margin status along with PTI was devised for the prediction of biochemical recurrence-free survival after RP, the predictive accuracies of the multivariate model that included and excluded PTI were $78.1 \%$ and $77.4 \%$, respectively, among the total patient population $(P=0.459)$ (Figure 2). Among the low-risk group, the accuracy of the predictive multivariate model with PTI included and excluded was $94.2 \%$ and $91.0 \%$, respectively $(P=0.268)$.

Table 2 Multivariate Cox proportional hazards model of the potential predictors for biochemical recurrence-free survival among the 534 total patients

\begin{tabular}{lccc}
\hline & HR & $95 \% \mathrm{Cl}$ & P value \\
\hline Age & 1.061 & $0.720-1.564$ & 0.763 \\
Preoperative PSA level & 1.168 & $0.884-1.544$ & 0.274 \\
Prostate volume & 0.994 & $0.979-1.011$ & 0.498 \\
Pathological Gleason score & 2.542 & $1.299-4.974$ & 0.006 \\
Extraprostatic extension of tumour & 1.998 & $1.045-3.820$ & 0.036 \\
Seminal vesicle invasion & 1.785 & $1.065-2.994$ & 0.028 \\
Surgical margin positivity & 1.027 & $0.640-1.649$ & 0.912 \\
Percentage of tumour involvement & 1.323 & $1.010-1.734$ & 0.042 \\
\hline
\end{tabular}

Abbreviations: $\mathrm{Cl}$, confidence interval; HR, hazard ratio; PSA, prostate-specific antigen. 

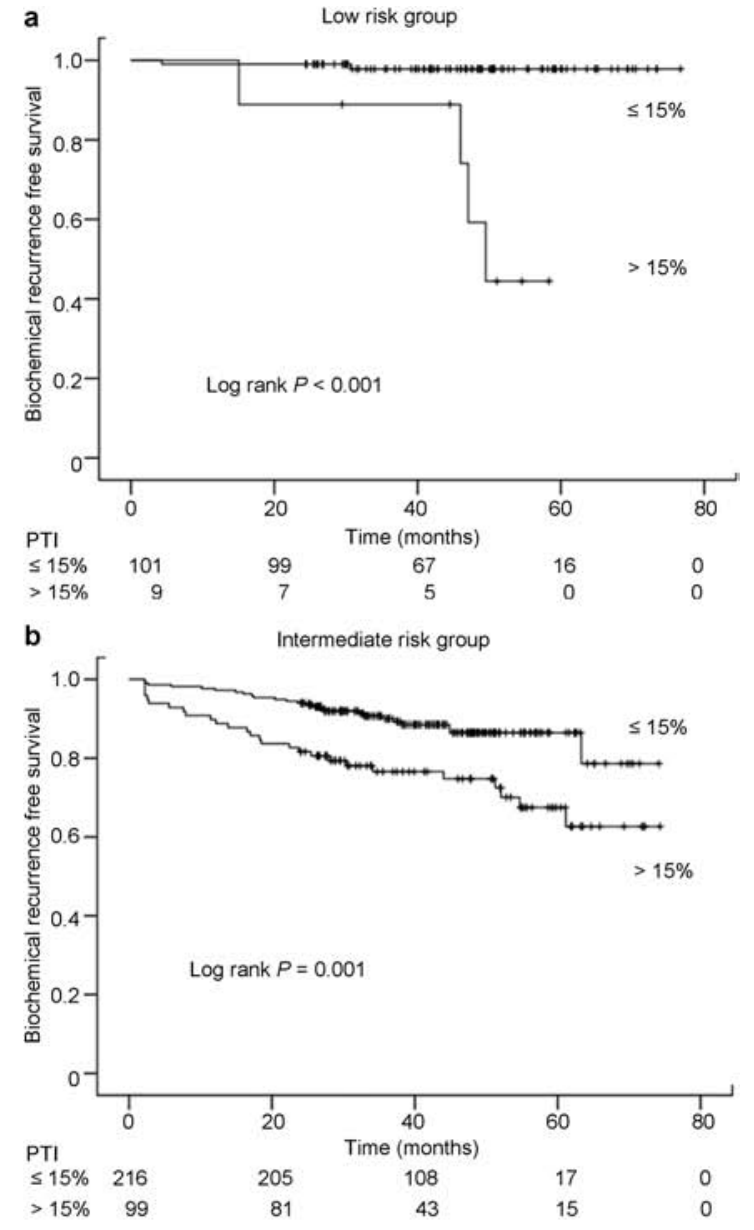

c

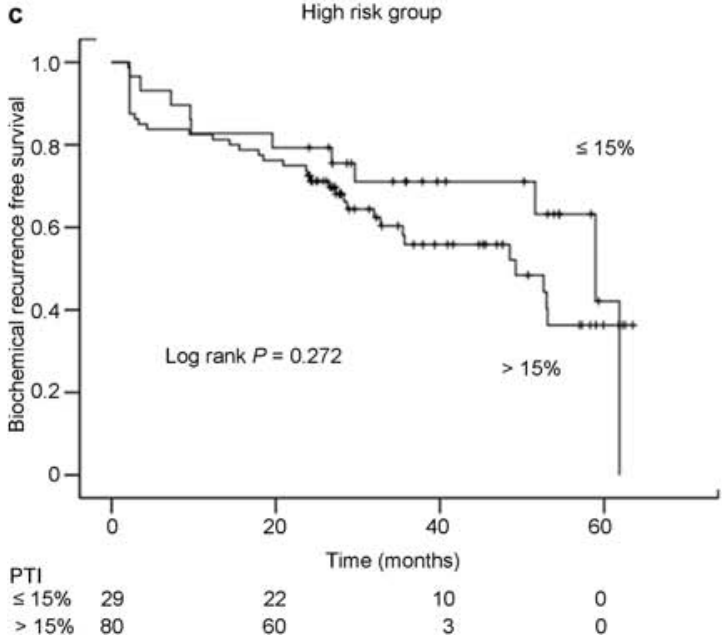

Figure 1 Kaplan-Meier curve demonstrating the estimates of biochemical recurrence-free survival according to percent of tumour involvement among (PTI) (a) low-risk group, (b) intermediate-risk group and (c) high-risk group.

\section{DISCUSSION}

In our study, PTI was an independent predictor of biochemical recurrence-free survival following RP among the total subject population. On the other hand, when patients were stratified according to disease risk groups as determined by preoperative PSA level and pathological Gleason score, PTI remained as an independent predictor of biochemical recurrence-free survival only among the low-risk group. In addition, the accuracy of the multivariate model devised for predicting biochemical progression by incorporating established prognostic variables was not significantly increased via the addition of PTI into the model among both the total patients and the low-risk group only. These findings indicate that PTI may not enhance the performance of an already optimized model for the prediction of biochemical progression after RP.

Other studies have previously examined the association between PTI assessed in an RP specimen and the outcome measures of pathological parameters and biochemical recurrence. Using grid morphometric technique, Humphrey et al. ${ }^{9,10}$ reported that the intraglandular tumour extent measured as carcinoma percentage correlated with disease progression following RP among patients with clinically localized prostate cancer. In addition, Carvalhal et al. ${ }^{11}$ and Ramos et al. ${ }^{12}$ observed that PTI was an independent predictor of the biochemical recurrence among patients who underwent RP for pathologically organ-confined disease. Furthermore, Rampersaud et al. ${ }^{13}$ reported that PTI remained significantly associated with disease progression following RP even after adjustment for multiple clinical and pathological characteristics for men with margin positive or extraprostatic tumour extension as well as those with pathologically organ-confined disease. Their observations suggested that PTI would be especially useful in the selection of candidates for adjuvant therapy among men with unfavourable pathological features of margin positivity or extraprostatic tumour extension. However, Epstein et al. ${ }^{14}$ in their study of clinically localized prostate cancer patients treated with RP, reported that both TV and PTI did not provide independent information beyond what was offered by other variables such as Gleason score. In addition, Uhlman et al. ${ }^{15}$ recently reported that PTI was a significant predictor of biochemical progression only among patients with a prostate less than $35 \mathrm{ml}$ in volume. Overall, the previous reports can be considered reflective of the ongoing controversy surrounding the actual value of PTI as a potential prognostic indicator in RP patients.

Unlike the previously reported studies, we stratified patients according to disease risk groups and observed that PTI was an independent predictor of biochemical progression only among patients with relatively less aggressive diseases of low risk. Notably, although PTI was associated with respective adverse pathological features of extraprostatic tumour extension, high $(\geqslant 7)$ Gleason score, seminal vesicle involvement and margin positivity along with preoperative PSA level, it was not an independent predictor of biochemical progression for the intermediate- and high-risk groups when adjusted for established prognostic parameters. Previously, Ramos et al. ${ }^{12}$ reported that the prognostic significance of TV or PTI would be obscured by a high probability of recurrence due to the tumour extending to the surgical margins or beyond prostate in patients with such disease. Such a report can be considered as being at least partially supportive of our results on disease risk groups. However, other studies have reported that PTI correlates with outcome following RP in patients with margin positivity or extraprostatic extension of tumour. ${ }^{13,18}$ Complicating the issue further, some groups have recently reported that the prognostic significance of PTI is only evident among patients with relatively smaller prostate ${ }^{15}$ The results of the present study suggest that the prognostic impact of PTI may vary according to overall disease aggressiveness. According to our results, the prognostic impact of PTI would be overwhelmed by the influence of other established prognostic factors in patients with diseases of intermediate or higher risk, as can be seen from the results of multivariate analyses. In our study, prostate size was controlled for in the various multivariate 
Table 3 Multivariate Cox proportional hazards model of the potential predictors for biochemical recurrence-free survival among each disease risk group

\begin{tabular}{|c|c|c|c|c|c|c|c|c|c|}
\hline & \multicolumn{3}{|c|}{ Low risk group $(\mathrm{n}=110)$} & \multicolumn{3}{|c|}{ Intermediate risk group $(\mathrm{n}=315)$} & \multicolumn{3}{|c|}{ High risk group $(\mathrm{n}=109)$} \\
\hline &,$I R$ & $95 \% \mathrm{Cl}$ & $\mathrm{P}$ value & $H R$ & $95 \% \mathrm{Cl}$ & $\mathrm{P}$ value & $\Pi R$ & $95 \% \mathrm{Cl}$ & $\mathrm{P}$ value \\
\hline Age & 1.021 & $0.963-1.079$ & 0.058 & 1.352 & $0.979-1.722$ & 0.386 & 1.530 & $0.972-2.088$ & 0.230 \\
\hline Preoperative PSA level & 1.762 & $0.946-3.282$ & 0.074 & 2.000 & $1.047-3.819$ & 0.033 & 1.028 & $0.771-1.369$ & 0.852 \\
\hline Prostate volume & 0.978 & $0.860-1.111$ & 0.723 & 0.971 & $0.945-0.997$ & 0.034 & 0.988 & $0.933-1.043$ & 0.100 \\
\hline Pathological Gleason score & - & - & - & 3.191 & $1.086-9.203$ & 0.025 & 2.298 & $1.065-4.958$ & 0.034 \\
\hline Extraprostatic extension & 1.836 & $1.006-3.353$ & 0.048 & 1.421 & $0.991-2.786$ & 0.054 & 2.346 & $1.260-3.433$ & 0.012 \\
\hline Seminal vesicle invasion & - & - & - & 1.451 & $0.904-3.244$ & 0.451 & 2.298 & $1.074-3.522$ & 0.034 \\
\hline Surgical margin positivity & 1.540 & $0.938-2.142$ & 0.654 & 1.075 & $0.523-2.279$ & 0.826 & 1.091 & $0.523-2.279$ & 0.816 \\
\hline Percentage of tumour involvement & 2.000 & $1.057-3.785$ & 0.033 & 1.421 & $0.818-2.934$ & 0.287 & 1.091 & $0.498-2.406$ & 0.828 \\
\hline
\end{tabular}

Abbreviations: $\mathrm{Cl}$, confidence interval; $\mathrm{HR}$, hazard ratio; PSA, prostate-specific antigen.

analyses performed, and we could not confirm the effect of prostate size on PTI as previously reported. ${ }^{15}$

When a potential predictive or prognostic marker is analysed for efficacy, it should be judged on its capacity to improve a pre-existing optimized predictive model rather than simply on its status as an independent variable. ${ }^{19}$ In our study, despite its status as an independent predictor, the inclusion of PTI in the multivariable models devised for predicting biochemical recurrence among the total patient and the low-risk groups, respectively, failed to enhance the predictive performances of the model. Therefore, our results suggest that PTI may not be a necessary component of routine pathological reports, as it did not offer additional prognostic information beyond the information provided by established classical prognostic factors, such as PSA, pathological Gleason score, pathological stage and surgical margin status.

Certainly, TV, as measured by a computerized morphometric analyser, would be a more accurate estimate of actual tumour burden in RP specimens than PTI. Recently, Sherwin et al. ${ }^{20}$ reported that TV measured as a percentage of prostate volume determined objectively via image analysis software, which can be considered computer-assessed PTI, correlated with the pathological features of extraprostatic tumour extension, positive surgical margin and seminal vesicle invasion. Still, to the best of our knowledge, the

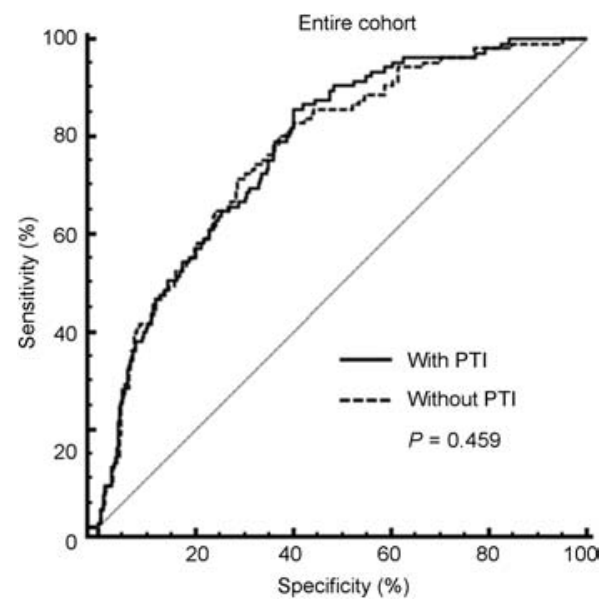

Figure 2 Receiver-operating characteristics curves of multivariate logistic regression model devised for the prediction of biochemical recurrence after radical prostatectomy (RP) with and without percentage of tumour involvement (PTI) among the total patients. superiority of computer-assessed parameters, as relevant to tumour size as a prognostic parameter for prostate cancer, over those assessed visually by pathologists, has yet to be validated via prospective study. Moreover, due to medical insurance issues in various countries along with other factors, the reporting of computerassisted TV measurement is not likely to become a standard practice in the near future. In the interim, additional studies are needed to investigate the prognostic impact of computer-assessed TV in prostate cancer.

The potential limitations of our study include the retrospective nature of study design. In addition, we could not control for intraor interobserver variability in assessing PTI. Also, lymph node status was not included as a variable in multivariate analyses due to large number of patients with unknown lymph node status. In addition, we could not analyse the extent of surgical margin. In Korea, PSA screening is not as widely applied as in Western countries. Accordingly, prostate cancers arising in Korean men tend exhibit poorer differentiation and more aggressive features compared with those observed in Western patients. ${ }^{21}$ We believe that such factors may well have contributed to the higher rate of positive surgical margin in our series compared with Western RP series. In addition, body mass index was not included in our analysis. Previously, we have reported that body mass index did not significantly enhance the ability to preoperatively predict pathological outcome and was not significantly associated with PSA outcome or with other objective pathological outcomes in Korean men undergoing RP, who are generally leaner than Western counterparts. $^{22}$ Due to lack of a long-term follow-up, the time to biochemical recurrence rather than cancer-specific survival was designated as primary endpoint in our study. Biochemical recurrence would currently be the most commonly applied parameter in analysing outcomes following RP. The results of our study may have been different with a larger number of subjects and a longer follow-up period.

\section{CONCLUSIONS}

Although PTI was observed to be a more significant prognostic factor among patients with low-risk disease than among those with higher risk diseases, the addition of PTI failed to enhance the predictive accuracy of the multivariate model, which incorporated various established prognostic factors, devised for the prediction of biochemical progression following RP. Such findings suggest that PTI may not be a useful prognostic indicator in RP patients. Further investigation with a larger cohort is needed to confirm our results and also to determine whether our results apply to other methods of measuring tumour size, such as TV assessed via computerized system. 
neg

Percentage of tumour involvement according to disease risk group

JJ Oh et al

832

AUTHOR CONTRIBUTIONS

JJO and SKH contributed to the study concept and design. JJO wrote the draft of the manuscript under the supervision of SKH, SEL and SSB. GYC conducted the pathological evaluation. SCL, CWJ, CYY, SJJ and YKR conceived of the study, participated in its design and coondination and helped to draft the manuscript. All authors read and approved the final manuscript.

COMPETING FINANCIAL INTERESTS

The authors declare no competing financial interests.

1 Stamey TA, Freida FS, McNeal JE, Redwine EA, Whittemore AS et al. Localized prostate cancer: relationship of tumor volume to clinical significance for treatment of prostate cancer. Cancer 1993; 71: 933-8.

2 Stamey TA, McNeal JE, Yemoto CM, Siqal BM, Johnston IM. Biological determinants of cancer progression in men with prostate cancer. J Am Med Assoc 1999; 281: 1395-400.

3 Nelson BA, Shappell SB, Chang SS, Wells N, Farnham SB et al. Tumour volume is an independent predictor of prostate-specific antigen recurrence in patients undergoing radical prostatectomy for clinically localized prostate cancer. BJU Int 2006; 97: 1169-72.

4 Chin FK, Briganti A, Jeldres C, Galling A, Erbersdobler A et al. Tumour volume and high grade tumour volume are the best predictors of pathologic stage and biochemical recurrence after radical prostatectomy. Eur J Cancer 2007; 43: 536-43.

5 Vollmer RT. Percentage of tumor in prostatectomy specimens: a study of American veterans. Am J Chin Pathol 2009; 131: 86-91.

6 Kikuchi E, Scardino PT, Wheeler TM, Slawin KM, Ohori M. Is tumor volume an independent prognostic factor in clinically localized prostate cancer? J Urol 2004; 172: $508-11$.

7 Merrill MM, Lane BR, Reuther AM, Chou M, Magi-Galluzzi C et al. Tumor volume does not predict for biochemical recurrence after radical prostatectomy in patients with surgical Gleason score 6 or less prostate cancer. Urology 2007; 70: 294-8.

8 Porten SP, Cooperberg MR, Carroll PR. The independent value of tumour volume in a contemporary cohort of men treated with radical prostatectomy for clinically localized disease. BJU Int2010; 105: 472-5.

9 Humphrey PA, Vollmer RT. Intraglandular tumor extent and prognosis in prostatic carcinoma: application of a grid method to prostatectomy specimens. Hum Pathol 1990; 21 : 799-804.
10 Humphrey PA, Walter PJ, Currin SM, Vollmer RT. Histologic grade, DNA ploidy, and intraglandular tumor extent as indicators of tumor progression of clinical stage B prostatic carcinoma. A direct comparison. Am J Surg Pathol 1991; 15: 116570.

11 Carvalhal GF, Humphrey PA, Thorson P, Man Y, Ramos CG et al. Visual estimate of the percentage of carcinoma is an independent predictor of prostate carcinoma recurrence after radical prostatectomy. Cancer 2000; 89: 1308-14.

12 Ramos CG, Roehl KA, Antenor JA, Humphrey PA, Catalonia WJ. Percent carcinoma in prostatectomy specimen is associated with risk of recurrence after radical prostatectomy in patients with pathologically organ confined prostate cancer. $J$ Urol 2004; 172: 137-40.

13 Rampersaud EN, Sun L, Moul JW, Madden J, Freedland SJ. Percent tumor involvement and risk of biochemical progression after radical prostatectomy. $J$ Ural 2008; 180: 571-6.

14 Epstein JI, Carmichael M, Martin AW, Walsh PC. Is tumor volume an independent predictor of progression following radical prostatectomy? A multivariate analysis of 185 clinical stage B adenocarcinomas of the prostate with 5 years of follow up. J Ural 1993; 149: 1478-81.

15 Uhlman MA, Sun L, Stackhouse DA, Polascik TJ, Mouraview V et al. Tumor percent involvement predicts prostate specific antigen recurrence after radical prostatectomy only in men with smaller prostate. J Urol 2010; 183: 997-1001

16 Alkhateeb S, Alibhai S, Flesher N, Finelli A, Jowett M et al. Impact of positive surgical margins after radical prostatectomy differs by disease risk group. J Ural 2010; 183: 145-50.

17 Cookson MS, Aus G, Burnett AL, Canby-Hagino ED, D'amico AV et al. Variation in the definition of biochemical recurrence in patients treated for localized prostate cancer: the American Urological Association Prostate Guidelines for Localized Prostate Cancer Update Panel report and recommendations for a standard in the reporting of surgical outcomes. J Ural 2007; 177: 540-5.

$18 \mathrm{Ku} \mathrm{JH}$, Moon KC, Che SY, Kwa C, Kim HH. Serum prostate-specific antigen value adjusted for non-cancerous prostate tissue volume in patients undergoing radical prostatectomy: a new predictor of biochemical recurrence in localized or locally advanced prostate cancer. Asian J Androl 2011; 13: 248-53

19 Kaftan MW. Judging new markers by their ability to improve predictive accuracy. J Nat/ Cancer Inst 2003; 95: 634-5.

20 Sherwin JC, Mirmilstein G, Pedersen J, Lawrentschuk N, Bolton D et al. Tumor volume in radical prostatectomy specimens assessed by digital image analysis software correlates with other prognostic factors. J Urol 2010; 183: 1808-14.

21 Song C, Bo JY, Lee MS, Hong SJ, Chung BH et al. Prostate cancer in Korean men exhibits poor differentiation and is adversely related to prognosis after radical prostatectomy. Urology 2006; 68: 820-4.

22 Lee SE, Lee WK, Jeong MS, Abdullajanov M, Kim DS et al. Is body mass index associated with pathological outcomes after radical prostatectomy in Korean men? BJU Int 2010; 107: 1250-5

Asian Journal of Andrology 